\title{
Entrapment of the Left Renal Vein: A Rare Cause of Proteinuria
}

\author{
Sik Lee ${ }^{\mathrm{a}, \mathrm{c}}$, Young Min Honga, Kyung Pyo Kanga, Won Kimª, \\ Sung Kwang Park ${ }^{\mathrm{a}}$, Jong Kwan Park ${ }^{\mathrm{b}}$
}

\section{To the Editor:}

A 26-year-old man visited our institution for evaluation of proteinuria. He intermittently presented with acute urinary retention. Other urological evaluations were unremarkable. Urinalysis exhibited trace proteins without hematuria. Renal duplex and enhanced computed tomography (CT) of the abdomen and pelvis were performed. The duplex ultrasound typically demonstrated aortomesenteric anatomy with entrapment of the left renal vein (LRV) (Fig. 1). The diameters of the LRV at the hilar and aortomesenteric portions were measaured as $11 \mathrm{~mm}$ and $1.2 \mathrm{~mm}$, respectively. The duplex ultrasound also indicated a significantly higher ratio of the peak systolic velocity (PSV) to the hilar renal vein PSV at the point of renal vein compression $(>6)$. This observation demonstrates the diminished volume of flow and dampened pulsatility upon spectral analysis of the left renal vein. Enhanced CT angiography revealed compression of the left renal vein between the aorta and the superior mesenteric artery (Fig. 2). The Nutcracker syndrome is defined as a rare cause of hematuria and flank pain caused by compression of the left renal vein between the aorta and the superior mesenteric artery where it passes at the point of bifurcation of the arteries [1]. The observed compression causes venous hypertension and the formation of renal venous collaterals leading to the subsequent rupture of the septum between the veins and the collecting system, and finally, resulting in hematuria and flank pain $[2,3]$. In this particular patient case, although rare, the nutcracker should be included as the potential cause of proteinuria in addition to hematuria in the absence of other renal diseases.

Manuscript accepted for publication June11, 2012

${ }^{a}$ Department of Internal Medicine, Renal Research Institute of Clinical Medicine, Chonbuk National University Medical School, Jeonju, Republic of Korea

${ }^{b}$ Department of Urology, Renal Research Institute of Clinical Medicine, Chonbuk National University Medical School, Jeonju, Republic of Korea ${ }^{\circ}$ Corresponding author: Sik Lee, Department of Internal Medicine, Chonbuk National University Medical School, 634-18, Keum-Am Dong, Jeonju, 561-712, Republic of Korea. Email: kidney@jbnu.ac.kr
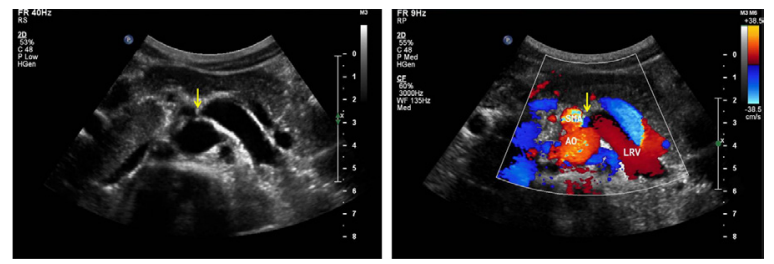

Figure 1. The duplex ultrasound typically demonstrated aortomesenteric anatomy with entrapment of the left renal vein (LRV).

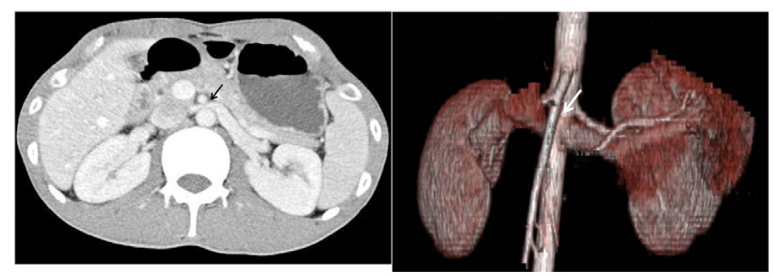

Figure 2. Enhanced CT angiography revealed compression of the left renal vein between the aorta and the superior mesenteric artery.

\section{References}

1. Jang YB, Kang KP, Lee S, Kim W, Kwak HS, Park SK. Posterior nutcracker phenomenon. Nephrol Dial Transplant. 2005;20(11):2573-2574.

2. Pytel A. Renal fornical hemorrhages: their pathogenesis and treatment. J Urol. 1960;83:783-789.

3. Rudloff U, Holmes RJ, Prem JT, Faust GR, Moldwin R, Siegel D. Mesoaortic compression of the left renal vein (nutcracker syndrome): case reports and review of the literature. Ann Vasc Surg. 2006;20(1):120-129. 